\title{
Fiber Optics and Photonics: European Heritage
}

\author{
Tomasz R. Woliński ${ }^{* 1}$ and Philip Russell ${ }^{2}$ \\ ${ }^{1}$ Faculty of Physics, Warsaw University of Technology, Koszykowa 75, 00-662 Warszawa, Poland \\ ${ }^{2}$ Max Planck Institute for the Science of Light, Guenther-Scharowsky Str. 1, 90158 Erlangen, Germany
}

Received March 30, 2010; accepted March 31, 2010; published March 31, 2010

\begin{abstract}
We present here a brief history and an editorial review of fiber optics and photonics including in particular European and Polish heritage.
\end{abstract}

Charles K. Kao, the "Master of light" received the 2009 Nobel Prize in Physics "for groundbreaking achievements concerning the transmission of light in fibers for optical communication". Kao worked in 1966 in Europe, at the Standard Telecomm Laboratories (UK), where he proposed that low-attenuation light propagation in optical fibers should, in principle, be possible, although his first demonstration showed rather high attenuation. He is viewed as one of the fathers of today's high-speed internet transmission. The problem to solve was to manufacture glass fibers without impurities. Kao had pointed to the right inorganic material, namely fused silica [1]. Since 1966, an intense worldwide search with the aim of producing glass fibers with low losses began. Low loss fibers were drawn in 1970 at the Corning Glass Company and by 1979 the losses were reduced to $0.2 \mathrm{~dB} / \mathrm{km}$. It should be noted that one of the first 4 fiber lines worldwide was installed in Lublin, Poland, in 1978 and was based on multimode fibers manufactured by a chemists group led by Andrzej Waksmundzki and Jan Wójcik of Maria Curie-Skłodowska University (MCSU) in Lublin. The group obtained in 1988 the Polish State Award for "outstanding achievements in optical fiber technology". The advantages of optical fibers had been recognized even earlier in Poland and a conference on optical fibers and their applications was organized in 1976 in Jablonna [2]. Nowadays, several terabits per second can be transmitted in a single fiber. The number of optical fiber cables being installed all over the world is increasing rapidly. Since the early 1990 s, the interest in polarization mode dispersion (PMD) has been constantly increasing. The main reason is that PMD broadens up the pulse of light in the fiber line as a result of residual linear birefringence that is present in a telecommunications fiber and therefore limits the bit rate transmitted in a fiber. Single-mode optical fiber used in telecommunications and its components can support one fundamental mode, which

\footnotetext{
*E-mail: wolinski@if.pw.edu.pl
}

consists of two orthogonal polarization modes. Ideally, the core of an optical fiber is perfectly circular, and therefore has the same index of refraction for both polarization states. However, mechanical and thermal stresses introduced during manufacturing result in asymmetries in fiber core geometry. This asymmetry introduces a small index of refraction differences for the two polarization, the property called birefringence, which in telecom fibers accumulates stochastically along propagation length. External mechanical stresses and environmental conditions (e.g. temperature variations) exacerbate the problem. This phenomenon limits the development of a high-speed telecommunications system and therefore there is an urgent need for PMD compensating in thousands of kilometers of the "old" telecommunications fibers already installed throughout the world.

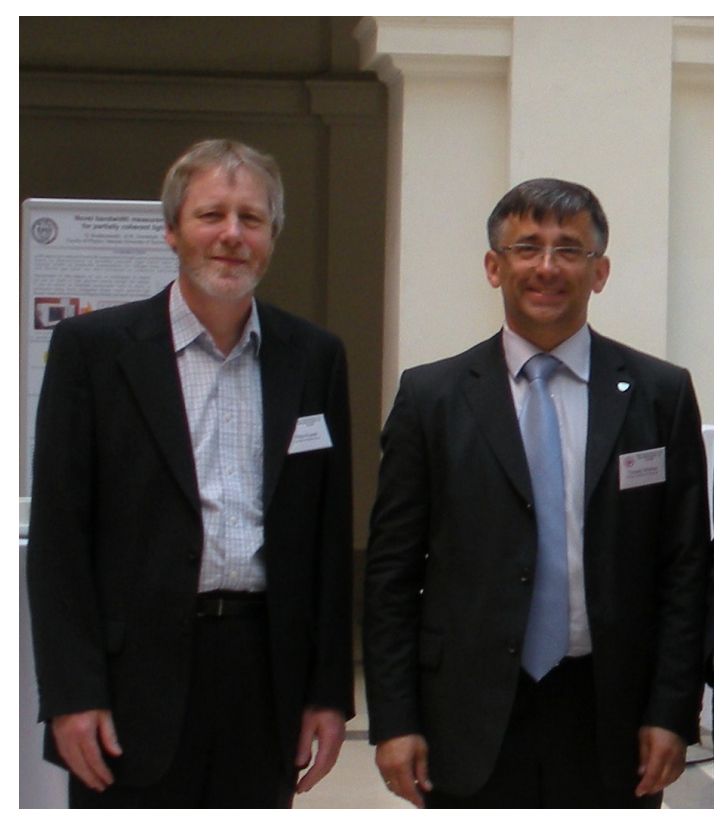

Fig. 1 Philip Russell (left) and Tomasz Woliński (right) at the Faculty of Physics Aula, Warsaw University of Technology, Warsaw, Poland during the Inauguration Symposium of the Photonics Society of Poland (31 May 2008). 
Photonic crystal fibers (PCFs) were proposed in 1991 by Philip Russell (presently at the Max Planck Institute for the Science of Light, Erlangen, Germany) and first manufactured in 1995 at the University of Southampton, and thereafter mainly at the University of Bath. They have attracted significant interest since the modification of their microstructure allows remarkable control of key optical properties such as dispersion, birefringence, nonlinearity, and both the position and width of the photonic bandgaps (PBGs) in the periodic "photoniccrystal" cladding [3]. In these fibers light can be guided by two different mechanisms: so-called index-guiding (similar to the classical waveguide effect based on a total internal reflection) and the photonic bandgap effect. PBG confinement is unique in allowing guidance even when the effective (average) refractive index of the microstructured cladding is higher than the refractive index of the core. Typically, this results in spectral windows of low loss interspersed with high loss regions.

The propagation characteristics in a PCF can be further dynamically tuned by filling the hollow channels with substances whose refractive indices can be controlled by external fields. Liquid crystals are a very good candidate for making tunable in-fiber devices because they exhibit very high electro-optic and thermo-optic effects. The first evidence of photonic band-gap tuning in a PCF filled with an LC was reported by a Danish-Swedish team in 2003 [4]. However, similar results were published also in Poland [5] and elsewhere. The importance of research activities in the field of photonic crystal fibers was distinguished in 2009 by the Foundation for Polish Science. Two of nine national rewards in the 2009 Master Program were granted to physicists dealing either with photonic crystal fibers (Wacław Urbańczyk) or with photonic liquid crystal fibers (Tomasz R. Woliński).

This special issue of the Photonics Letters of Poland is devoted to fiber optics and photonics. Its core comes from papers presented at the 12 th Polish conference on optical fibers and their applications TAL-2009 held in Krasnobród, Poland in October 2009. The acronym TAL stands for the proper conference name: "Technology and Application of Lightguides" and was proposed in 1996 by Maksymilian Pluta, who had been President of the Polish Chapter of SPIE for many years. However, the first Polish National Symposium on Optical Fibers and their applications took place in Jablonna near Warszawa (in 1976) where the first Polish optical fibers manufactured from multicomponent glasses by a group of chemists led by Andrzej Waksmundzki of Maria Curie-Sklodowska University (MCSU) in Lublin were presented. This first

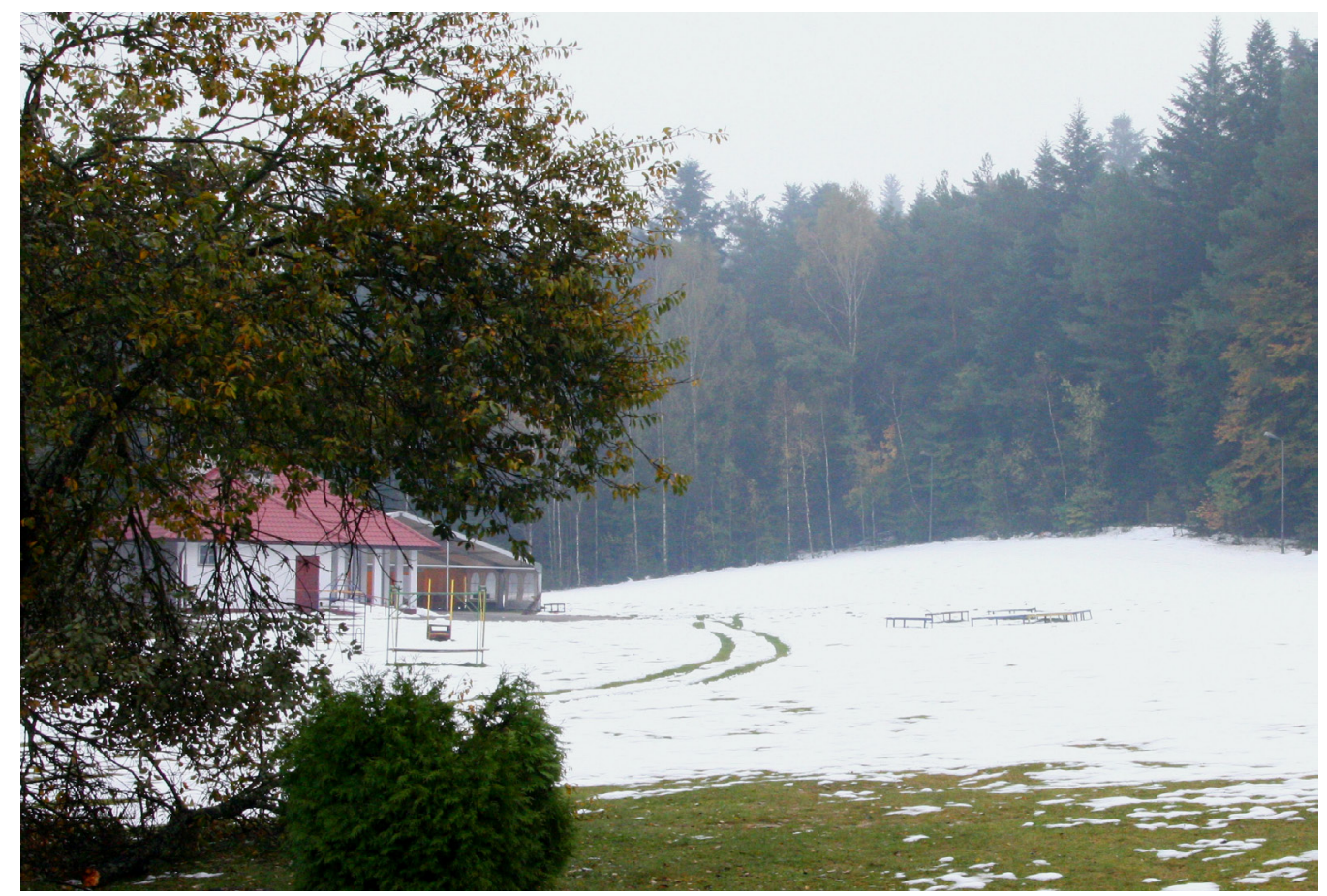

Fig. 2 12th Polish Conference on Optical Fibers and Their Applications (TAL) held in October 2009: a beautiful conference venue in Krasnobród (Roztocze, Poland) in the winter scenario. 
national event on optical fibers has been continued by two groups working on optical fiber technology: MCSU Laboratory of Optical Fibers in Lublin and a similar group in Białystok. Since 1996 TAL conferences have been organized by both leading centers and alternately in Krasnobród and Bialowieża, under the auspices of the Photonics Society of Poland (formerly Polish Chapter of SPIE), the Committee for Electronics and Telecommunications of the Polish Academy of Sciences, and the Polish Committee for Optoelectronics. To the leaders and the chairmen of the TAL conferences presently belong: Jan Dorosz, Zdzisław Jankiewicz, Jan Rayss, Wiesław Woliński, Jan Wójcik, and Waldemar Wójcik. The purpose of these conferences is to provide a forum for both senior and young scientists, generally from all over Poland but with participation of invited foreign specialists in the field, to present and discuss current research problems and activities in technology, physics and applications of optical fibers.

The diversity of the work reported here in the 17 papers covers a large spectrum of technology, theory and characterization of classical and microstructured fibers.
Special attention is devoted to the materials aspect of technology, sensor applications of optical fibers as well as optics and micro-optics for optical fiber technology applications. This includes research activities on different types of photonic crystal fibers based on fused silica, multicomponent glasses, plastic microstructured fibers, but also photonic crystal fibers infiltrated with nematic and chiral nematic liquid crystals.

\section{References}

[1] K.C. Kao, G.A. Hockham: Dielectric-fibre Surface Waveguides for Optical Frequencies, Proc. IEE 113, 1151-1158 (1966).

[2] First conference on optical fibers and their applications in 1976 in Jablonna was initiated by J. Groszkowski, B. Paszkowski, A. Smoliński, Z. Szpigler, and A. Waksmundzki.

[3] P. St. J. Russell, "Photonic-Crystal Fibers," J. Lightwave Technol. 24, 4729-4749 (2006).

[4] T. Larsen, A. Bjarklev, D. Hermann, and J. Broeng, "Optical devices based on liquid crystal photonic bandgap fibres," Opt. Express 11, 2589-2596 (2003).

[5] T. R. Wolinski, K. Szaniawska, K. Bondarczuk, P. Lesiak, A.W. Domanski, R. Dabrowski, E. Nowinowski-Kruszelnicki, J. Wojcik "Propagation properties of photonic crystals fibers filled with nematic liquid crystals", Opto-Electronics Rev. 13(2), 59-64 (2005). 